\title{
Metodologia de Otimização para Alocação Ótima de Medidores em Sistemas de Distribuição de Energia Elétrica *
}

\author{
Elisa Oliveira* Luíza M. Fortes ${ }^{* *}$ Leonardo W. de Oliveira ${ }^{* * *}$ \\ Edimar J. de Oliveira ${ }^{* * *}$ Denise S. de Melo ${ }^{\dagger}$ \\ Janaína G. de Oliveira ${ }^{\ddagger}$ Ana Flávia P. da Costa ${ }^{\S}$ \\ * Faculdade de Engenharia Elétrica, Universidade Federal de Juiz de \\ Fora, MG, (e-mail: elisa.oliveira@engenharia.ufjf.br). \\ ** Faculdade de Engenharia Elétrica, Universidade Federal de Juiz de \\ Fora, MG, (e-mail: mautone.luiza@engenharia.ufjf.br) \\ *** Faculdade de Engenharia Elétrica, Universidade Federal de Juiz de \\ Fora, MG, (e-mail: leonardo.willer@ufjf.edu.br) \\ **** Faculdade de Engenharia Elétrica, Universidade Federal de Juiz de \\ Fora, MG, (e-mail: edimar.oliveira@ufjf.edu.br) \\ † Faculdade de Engenharia Elétrica, Universidade Federal de Juiz de \\ Fora, MG, (e-mail: denise.melo@engenharia.ufjf.br) \\ ${ }_{\ddagger}$ Faculdade de Engenharia Elétrica, Universidade Federal de Juiz de \\ Fora, MG, (e-mail: janaina.oliveira@ufjf.edu.br) \\ $\S$ Faculdade de Engenharia Elétrica, Universidade Federal de Juiz de \\ Fora, MG, (e-mail: ana.procopio@engenharia.ufj.br)
}

\begin{abstract}
The present paper proposes an optimization approach for the optimal placement of measurers in electrical energy distribution systems with the purpose of improving the state estimation process by using the available measurements. For that purpose, the integer metaheuristic known as Artificial Immune Systems is used to determine the best network points to place measurers under the observability standpoint. In order to perform the state estimation, the weighted least square method of literature is applied. Two case studies involving two widespread distribution networks for planning and operation studies are presented to assess the proposed approach.

Resumo: O presente trabalho propõe um método de otimização para a alocação ótima de medidores em sistemas de distribuição de energia elétrica, visando à melhoria do processo de estimação de estados a partir das medidas disponíveis. Para tanto, utiliza-se a técnica metaheurística inteira denominada Sistemas Imunológicos Artificiais para determinar os melhores pontos da rede sob o ponto de vista da observabilidade para a localização de medidores. Para realizar a estimação de estados, utiliza-se o método dos mínimos quadrados ponderados da literatura. Dois estudos de casos envolvendo duas redes de distribuição amplamente investigadas em estudos de planejamento e operação são apresentados a fim de avaliar o método proposto.
\end{abstract}

Keywords: State Estimation; Optimization; Sistribution Systems; Optimal allocation; Artificial Immune System.

Palavras-chaves: Estimação de Estados; Otimização; Sistemas de Distribuição; Alocação ótima; Sistema Imune Artificial.

\section{INTRODUÇÃO}

Nas últimas décadas, o desenvolvimento tecnológico levou ao crescimento dos Sistemas Elétricos de Potência (SEP), surgindo as interligações com sistemas vizinhos. Nesta situação, é nescessário um monitoramento mais aprimorado e eficiente dos Centros de Controle e Supervisão (CCS) para que a rede seja operada de maneira eficiente e confiável (Abbasi and Seifi, 2013).

\footnotetext{
* Os autores deste artigo agradecem ao apoio da FAPEMIG, CNPq, CAPES, INERGE, e ao Grupo de Otimização GOPT.
}

Para supervisionar o sistema foi desenvolvida a técnica de Estimação de Estados (EE) (Schweppe and Rom, 1970) cujo objetivo é fornecer a estimativa do estado operativo da rede, ou seja, tensões nodais e fluxos de potência, usando um conjunto mínimo de medidas em tempo real obtidas através dos sistema de monitoramento SCADA e Unidades de Medição Fasorial (UMF) (Qing et al., 2015) (Yang et al., 2012).

A implementação de técnicas de estimação de estados minimiza o investimento em equipamentos de medição e infra-estrutura de comunicação, que seriam necessários 
para obter de forma direta o mesmo conjunto de medidas (Oliveira, 2016). Por esse motivo, a EE tem sido considerada como uma ferramenta básica de apoio ao operador do sistema.

Com o crescimento do Sistema de Distribuição de Energia (SDE), deu-se início em estudos de monitoramento em tempo real da rede de distribução (Schweppe and Rom, 1970). Dentro desta conjuntura, a técnica de EE é fundamental para Sistemas de Genrencimentos de Energia (SGE), já empregados em controle de redes de transmissão (Singh et al., 2009), e com a atual automação da rede elétrica requer-se a extenção dos EE para redes de distribuição (Ahmad et al., 2019).

No entanto, um dos maiores desafios da aplicação do processo de EE para o SDE é a escassez de medições reais extraídas da rede por meio de medidores (Korres and Manousakis, 2012). Com isso, na literatura existe técnicas de modelagem de carga do sistema para gerar peseudomedidas. Os EE utilizam dessas técnicas para contornar o problema de observabilidade da rede de distribuição (Wang and Schulz, 2004) (Chilard et al., 2009). Dessa forma, os estudos de Estimação de Estados em Sistemas de Distribuição (EESD) encontra-se aberto para pesquisas.

Dentre os estudos de estimação de estado destaca-se o método, já consolidado para sistemas de transmissão e redes de distribuição, apresentado por Singh et al. (2009). O estudo demonstra que o Método dos Mínimos Quadrados (MMQ), do inglês Weighted Least Squares (WLS), é viável para aplicação no SDE.

O estudo realizado por Sousa et al. (2018) apresenta um estimador sequencial com o intuito de possibilitar melhorias nos valores estimados para variáveis de estado do sistema. Já o recente trabalho apresentado por Ahmad et al. (2019) propõe um EE baseado em Rede Neural Artificial (RNA) que tem a capacidade de estimar diretamente o estado do sistema, e também estima pseudo-medidas com alta precisão. Destaca-se também o estudo proposto por Majidi et al. (2017) que utiliza dados fornecidos por Unidades de Medição de Micro-Fasores ( $\mu$ PMUs) as redes inteligentes de distribuição para uma nova e forte EESD resolvendo um conjunto de equações lineares sem qualquer processo iterativo, onde o vetor de correntes de injeção é esparso porque qualquer corrente de carga/gerador é insignificante quando comparado com a corrente total injetada da rede externa para a rede de distribuição.

Por fim, o estudo elaborado por Duque et al. (2017), que apresenta um Fluxo de Potência Ótimo Estendido (EOPF) para EE em SDE, destaca-se entre os trabalhos citados anteriormente por aplicar um procedimento de otimização para EE sem a necessidade de dados de carga históricos precisos, o que se encaixa no contexto de falta de medidas em SDE típicas.

Como o monitoramento total de um SDE é ainda economicamente inviável (Chilard et al., 2009), devido ao custo de UMFs e Medidores Inteligentes (MI), diversos estudos são encontrados na literatura com o objetivo de determinar lugares adequados para a instalação desses equipamentos. O estudo realizado por Moradi-Sepahvand et al. (2019), desenvolve um novo modelo para alocação ideal de uma combinação de UMFs monofásicas e trifásicas em estrura assimétrica de distribuição inteligente para observação completa. O modelo é formulado e apresentado como um Programa Linear Inteiro Binário (PLIB). Foram aplicadas duas retrições inovadoras para reduzir o número de UMFs.

No recente estudo realizado por Duque et al. (2017), que utiliza o E-OPF para EE, é possível verificar que existe uma eficiência computacional maior aplicando-se uma técnica de busca para determinar os locais de UMF que maximizam o desempenho do EE. No estudo são testados o aumento de um para dois, chegando até quatro UMFs, o que significa um aumento na redundância de medições.

Tendo em vista a importância e a complexidade do problema de estimação de estados em redes de distribuição de energia elétrica, aliada à relevância de uma decisão estratégica sobre a localização de medidores na rede, o presente trabalho apresenta um proposta de otimização para resolver este problema. Mais especificamente, uma técnica meta-heurística de otimização inteira é proposta para a determinação dos pontos de monitoramento, considerandose diferentes cenários quanto ao número de equipamentos disponíveis. Para avaliar o método proposto, dois sistemas da literatura são utilizados nos estudos de casos.

\section{FORMULAÇÃO DO PROBLEMA DE EE}

O problema de estimação de estados baseado no método dos mínimos quadrados ponderados é modelado por Wood et al. (2013). O vetor de medições pode ser equacionado como:

$$
Z=h(x)+e
$$

Em que:

$Z \quad$ vetor de medições;

$h(x)$ vetor de equações não lineares referente ao tipo de medição;

$x \quad$ variáveis de estado; $\mathrm{e}$

$e \quad$ vetor de erros associados às medições, considerado com distribuição gaussiana e média zero para o estimador WLS.

O problema de EE é de natureza não linear devido também à não linearidade de $h(x)$ e necessita, portanto, de métodos numéricos iterativos para sua resolução. A função objetivo consiste na minimização dos erros ou resíduos ponderados associados às medições, ou seja, na diferença entre as medidas e seus respectivos valores calculados através das equações $h(x)$. Desta forma, a formulação da função objetivo é apresentada em (2).

$$
\begin{aligned}
F O B=\min & \left(\frac{1}{2} \sum_{i=1}^{m} \frac{\left[Z_{i}-h_{i}(x)\right]^{2}}{\sigma_{i}^{2}}\right) \\
= & \min \left(\frac{1}{2}[Z-h(x)]^{\prime} R^{-} 1[Z-h(x)]\right)
\end{aligned}
$$

Em que:

$\sigma_{i} \quad$ variância da medida i; e

$R$ matriz diagonal de corvariância das medidas.

A matriz $R$ apresenta os pesos de cada medição, de modo que medições com grandes incertezas associadas possam 


\section{METODOLOGIA PROPOSTA}

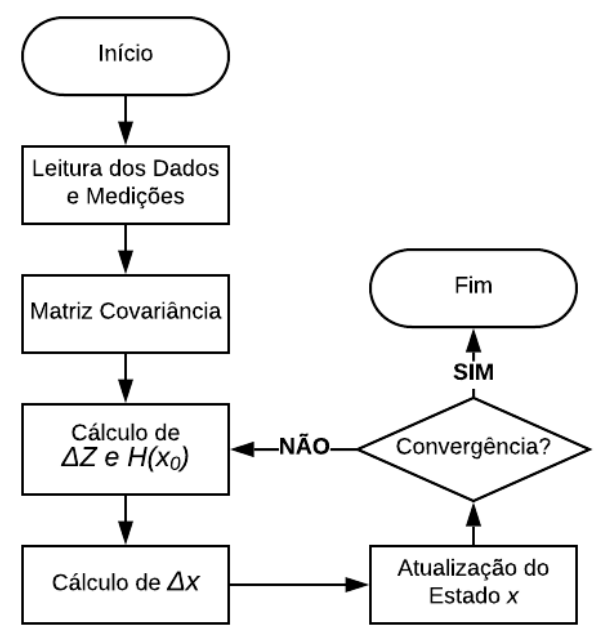

Figura 1. Fluxograma do estimador de estados WLS.

impactar menos no processo de EE, pois apresentam maiores valores de $\sigma_{i}$.

A resolução do problema de minimização relativo à EE é obtida através de linearização da equação em (2). Com isto, pode-se obter os passos para as variáveis de estado $x$ durante o processo iterativo de resolução, conforme (3).

$$
\Delta x=\left[H^{\prime}\left(x_{0}\right) R^{-1} H\left(x_{0}\right)\right]^{-1}\left[H^{\prime}\left(x_{0}\right) R^{-1} \Delta Z\right]
$$

Em que:

$$
\begin{gathered}
H\left(x_{0}\right)=\frac{\delta h(x)}{\delta x} \\
\Delta Z=Z-h\left(x_{0}\right)
\end{gathered}
$$

$H\left(x_{0}\right) \quad$ matriz Jacobiana das equações $h\left(x_{0}\right)$ no ponto $x_{0}$ cuja dimensão é dada pelo número de medições, igual ao número de equações, pelo número de variáveis de estado;

$H^{\prime}\left(x_{0}\right)$ matriz $H\left(x_{0}\right)$ transposta; e

$\Delta Z \quad$ vetor de erros absolutos entre as medidas e seus respectivos valores calculados por meio das equações $h(x)$ correspondentes, no ponto $x_{0}$.

As variáveis de estado são então atualizadas durante o processo iterativo a partir de seus passos conforme calculados em (3). A atualização do ponto $x_{0}$ para o ponto $x_{1}$ subsequente neste processo é formulada em (6).

$$
x_{1}=x_{0}+\Delta x
$$

O processo de estimação de estados alcança a convergência quando o máximo passo $\Delta x$ é menor que uma tolerância pré-especificada. O fluxograma desse processo é apresentado na Figura 1. O método iterativo consiste, portanto, na atualização dos vetores de erros absolutos entre as medidas e respectivos valores calculados, da matriz Jacobiana das equações de $h(x)$, dos passos $\Delta x$ e das variáveis de estado.

\subsection{Sistema Imunológico Artificial}

O método proposto no presente trabalho baseia-se na aplicação da técnica meta-heurística Sistema Imunológico Artificial (SIA) para determinar as localizações ótimas de medidores de tensão em SDE. O objetivo é minimizar o erro do processo de EE através de uma localização estratégica de medidores. Para tanto, aplica-se o algoritmo de otimização clonal, denominado CLONALG, utilizado em (De Castro and Von Zuben, 2002), (De Oliveira et al., 2014), cujos fundamentos são descritos de forma sucinta nesta seção.

O sistema imunológico artificial (Castro, 2009) é baseado no sistema imune natural adaptativo, inspirado em mecanismos avançados de identificação de agentes infecciosos. Em um problema de otimização, como o tratado no presente trabalho, o objetivo é identificar o ponto de mínimo ou máximo de uma função objetivo. Para tanto, o SIA dispõe dos mecanismos evolutivos descritos na Subseção 3.3 .

\subsection{Aplicação Proposta}

Na aplicação proposta, cada indivíduo do SIA corresponde a uma solução candidata do problema de alocação ótima de medidores em SDE. Para tanto, a codificação do indivíduo é feita de forma decimal, em que cada uma das $L$ posições de uma solução candidata recebe o número da barra candidata onde o medidor será alocado. Por exemplo, no vetor $B$ cand a seguir, associado a um sistema hipotético com cinco barras candidatas, a solução codificada determina medidores na segunda e na quarta barras candidatas.

$$
\text { Bcand }=\left[\begin{array}{ll}
2 & 4
\end{array}\right]
$$

\subsection{Algoritmo Proposto}

O algoritmo proposto em (De Oliveira et al., 2014), em que os fundamentos da técnica SIA (De Castro and Von Zuben, 1999) (De Castro and Von Zuben, 2000) são aplicados a problemas de otimização combinatória, e cujo fluxograma é mostrado na Figura 2, é aplicado no presente trabalho, sendo seus passos resumidos na sequência.

Passo 1: Geração inicial de anticorpos do repertório $P^{*}$ : processo aleatório de obtenção do conjunto inicial de soluções candidatas ao problema de alocação ótima de medidores. Destaca-se que $P^{*}$ representa uma matriz $[N A b \times$ $L]$, onde $N$ é o número de anticorpos e $L$ é o número de atributos de cada anticorpo. Ao final deste passo, a população atual $P$ recebe $P^{*}$.

Passo 2: Avaliação da afinidade $f$ dos anticorpos de $P$ : a afinidade de um anticorpo é dada pelo inverso do erro do estimador de estados WLS.

Passo 3: Seleção dos melhores anticorpos de $P$ : neste passo, os melhores anticorpos de $P$ são selecionados para compor o repertório $P n$.

Passo 4: Clonagem dos anticorpos de Pn: os anticorpos previamente selecionados em $P n$ são clonados, formando a população $C$. 


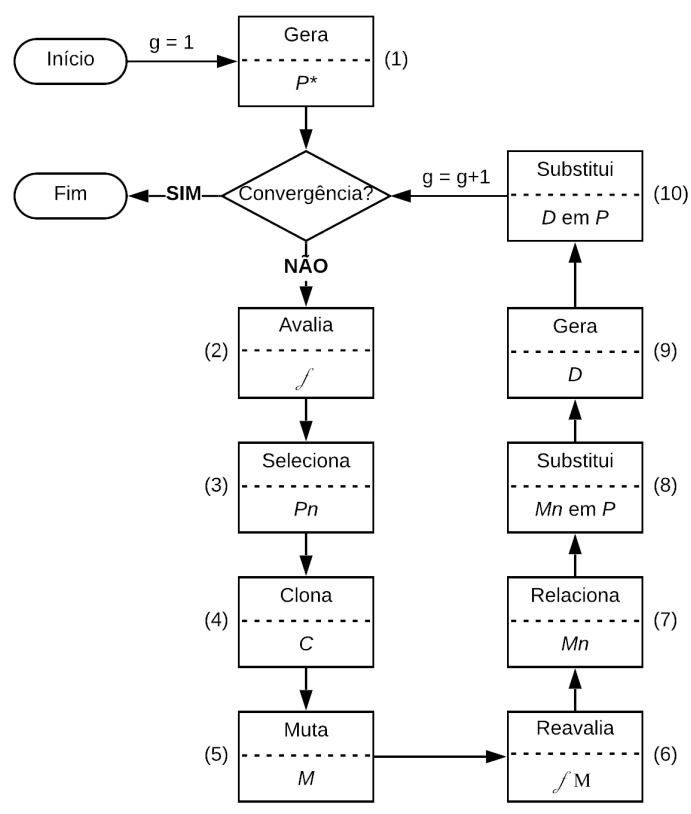

Figura 2. Fluxograma do algoritmo CLONR.

Passo 5: Hipermutação: neste passo, ocorre o processo de mutação dos anticorpos do repertório $C$, formando o conjunto de clones maduros $M$.

Passo 6: Avaliação da afinidade $f M$ dos anticorpos de $M$.

Passo 7: Seleção dos melhores anticorpos de $M$ : ocorre o processo descrito no Passo 3 formando a população $M n$.

Passo 8: Substituição dos piores anticorpos de $P$ pelos anticorpos de $M n$.

Passo 9: Edição de receptores: gera-se uma quantidade " $d$ ", pré-definida, de anticorpos, de modo aleatório, formando o conjunto $D$. Estes anticorpos substituem os " $d$ " anticorpos com menor afinidade da população $P$.

Passo 10: Substituição dos anticorpos de $P$ pelos anticorpos de $D$.

Após o Passo 10, o contador de geração (g) é incrementado e o critério de convergência é avaliado. Este critério é satisfeito quando pelo menos uma das seguintes condições é verdadeira: (i) o número de gerações atinge um valor limite dado por gmax; (ii) a melhor solução do repertório $P$ permanece inalterada durante um número de gerações dado por gstop. Caso não ocorra convergência o algoritmo retorna ao Passo 2.

\section{ESTUDO DE CASOS}

Esta seção apresenta os testes com os sistemas de 16 (Civanlar et al., 1988) e 33 (Baran and Wu, 1989) barras para avaliar a metodologia proposta. Para este propósito, o método WLS da literatura foi desenvolvido para avaliar a influência da localização do medidor de tensão, onde a localização ótima do candidato é definida pela técnica SIA visando minimizar o erro do EE.

O modelo não linear de rede de energia monofásica é considerado nos testes. Embora as cargas de um SDE sejam desequilibradas, os testes com um modelo monofásico podem mostrar a eficácia da abordagem proposta no problema de alocação de medidores para melhoria da estimativa de estado.

Neste trabalho, uma faixa de $-40 \%$ a $+40 \%$ é considerada para dados históricos de cargas de energia. Os testes WLS são realizados com medições de módulo e ângulo de fase na subestação (SE), além de dados históricos para cargas ativa e reativa. Os erros nas medidas dos medidores de tensão são de 0,4\% como em (Gomez-Exposito and Abur, 2004). Os resultados estimados do WLS são comparados com os valores reais correspondentes obtidos a partir do Fluxo de Potência Convencional (FPC) considerando pico de carga. Portanto, os resultados do FPC para módulo e ângulo de fase das tensões são considerados como os valores reais para avaliar o WLS. As simulações foram realizadas em um processador Intel Core i7-88550U de $1.80 \mathrm{GHz}$ com $8 \mathrm{~GB}$ de RAM. O software MATLABR foi utilizado para desenvolver o modelo proposto e realizar simulações dos estudos de casos apresentados.

A Tabela 1 apresenta os parâmetros do SIA utilizados, em que $\beta$ é o parâmetro de controle no processo de clonagem; $\rho$ é probabilidade de mutação dos anticorpos.

Tabela 1. Parâmetros do algoritmo.

\begin{tabular}{ccccccc}
$N A b$ & $\beta$ & $\rho$ & $d$ & gmax & gstop & $n$ \\
\hline 30 & 20 & 1 & 1 & 30 & 15 & $20 \%$ \\
\hline
\end{tabular}

A topologia do sistema teste de $23 \mathrm{kV}$ de 16 barras, modificado conforme Duque et al. (2017) para estudos de EE, é ilustrada na Figura 3. A solução do FPC para avaliar o WLS é dada na Tabela 2. O módulo e o ângulo de fase da tensão da SE são definidos como 1.0 pu e zero, respectivamente.

As simulações consideram a alocação de um até quatro medidores visando melhorar a estimativa de estado e avaliar o efeito de redundância de medição. A decisão de alocação através da metodologia proposta é comparada com a busca exaustiva por todas as soluções possíveis, a fim de avaliar o método proposto. A Tabela 3 apresenta o resultado do caso base para os sistemas de 14 e 33 barras, ou seja, considerando um único medidor na $\mathrm{SE}$ e peseudomedidas de $P$ e $Q$ em todas as barras.

Tabela 2. Resultado fluxo de potência convencional.

\begin{tabular}{ccc} 
Barra & Tensão(pu) & Ângulo(rad) \\
\hline 1 & 1,0000 & 0 \\
2 & 0,9544 & $-0,0168$ \\
3 & 0,9142 & $-0,0339$ \\
4 & 0,9473 & $-0,0217$ \\
5 & 0,9461 & $-0,0219$ \\
6 & 0,8374 & $-0,0559$ \\
7 & 0,8568 & $-0,0483$ \\
8 & 0,8231 & $-0,0586$ \\
9 & 0,8986 & $-0,0377$ \\
10 & 0,8500 & $-0,0529$ \\
11 & 0,8101 & $-0,0632$ \\
12 & 0,8188 & $-0,0596$ \\
13 & 0,8044 & $-0,0661$ \\
14 & 0,8028 & $-0,0668$ \\
\hline
\end{tabular}




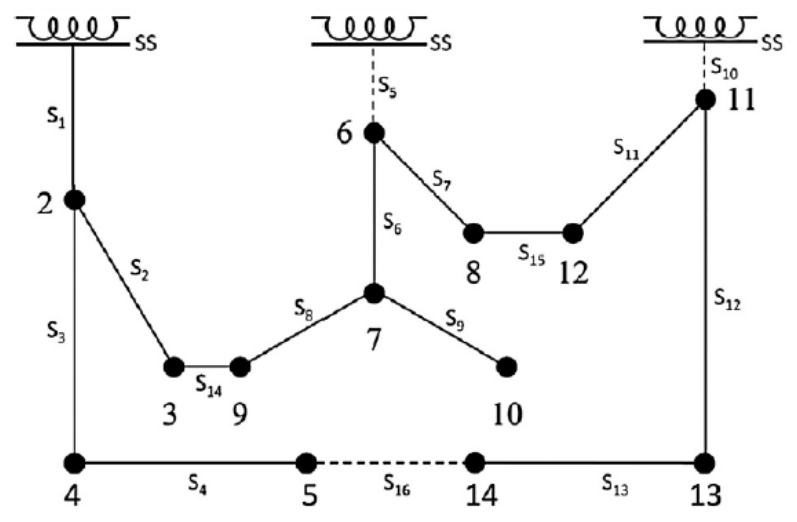

Figura 3. Topologia do sistema de 16 barras modificado.

\subsection{Simulação Sistema 16 Barras}

Esta simulação considera a minimização erro de EE $(F O B)$ para melhorar o desempenho do estimador. A Tabela 4 mostra cada termo e a $F O B$ para a análise de '1', '2', '3', e '4' medidores otimamente alocados e sua respectiva comparação as simulações feitas por buscas exaustivas, nas mesmas condições quanto ao número de medidores, na Tabela 5. Verifica-se que o EE aplicado com a solução proveniente da técnica SIA é tão preciso quanto o estimador atuando nas soluções obtidas por buscas exaustivas, uma vez que o SIA indicou as mesmas alocações. A alocação de quatro medidores é a melhor solução do ponto de vista da $F O B$, conforme esperado, devido ao aumento na redundância de medições.

\subsection{Simulação Sistema 33 Barras}

O sistema de teste de 33 barras é amplamente investigado na literatura e tem os dados encontrados em (Baran and Wu (1989)). A configuração original é composta por uma subestação e 32 ramais de distribuição, conforme Figura 4. A tensão e a potência base são iguais a $12,66 \mathrm{kV}$ e $10 \mathrm{MVA}$, respectivamente. Duas simulações são realizadas: (i) EE a partir da solução de alocação da técnica SIA; e (ii) EE aplicado à solução da busca exaustiva. As Tabelas 6 e 7 mostram os resultados obtidos para número de de ' 1 ' a '4' medidores. O melhor desempenho do estimador é obtido com '4' medidores, conforme esperado. A técnica SIA se iguala ao método de enumeração exautiva para '1', '2' e '4' medidores, porém, difere para '3' medidores.

Tabela 3. Resultado Caso Base.

\begin{tabular}{lc} 
Sistema & FOB $(\%)$ \\
\hline 14 barras & 17,5047 \\
33 barras & 24,2994 \\
\hline
\end{tabular}

Tabela 4. Resultados SIA.

\begin{tabular}{lcccc} 
Medidores & 1 & 2 & 3 & 4 \\
\hline Localização & 13 & $13-14$ & $12-13-14$ & $2-12-13-14$ \\
FOB $(\%)$ & 16,5875 & 16,4159 & 16,3754 & 16,2102 \\
\hline
\end{tabular}

Tabela 5. Resultados Busca Exaustiva.

\begin{tabular}{lcccc} 
Medidores & 1 & 2 & 3 & 4 \\
\hline Localização & 13 & $13-14$ & $12-13-14$ & $2-12-13-14$ \\
$F O B(\%)$ & 16,5875 & 16,4158 & 16,3754 & 16,2102 \\
\hline
\end{tabular}

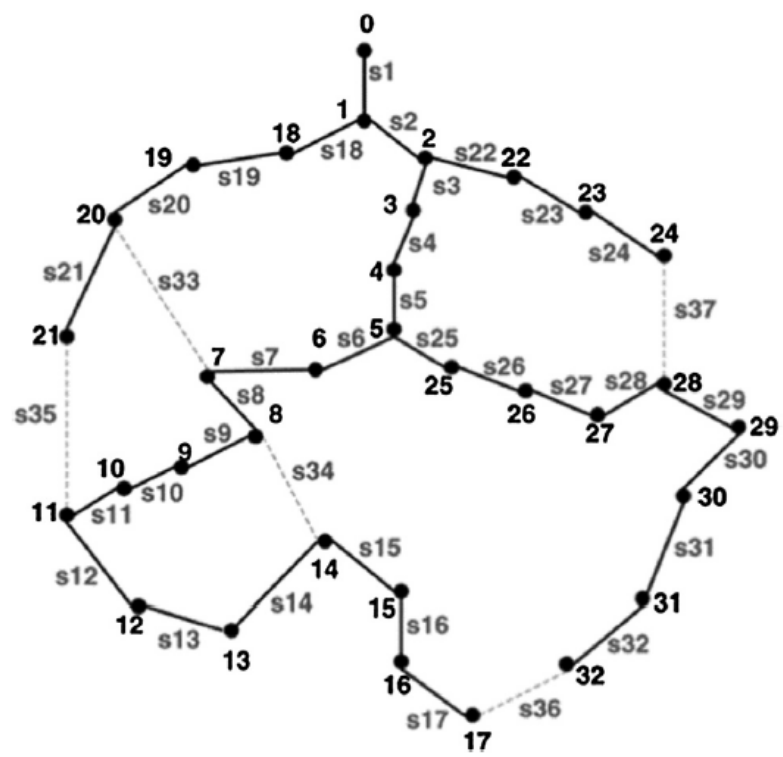

Figura 4. Topologia do sistema de 33 barras.
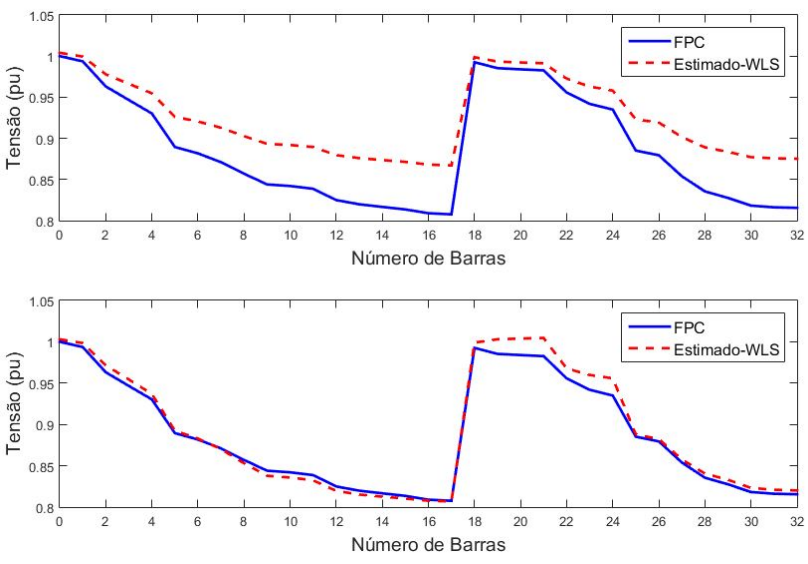

Figura 5. Módulo de tensão sem medidor $\times 4$ medidores.

No entanto, a solução obtida para '3' medidores ainda é de boa qualidade, haja vista sua proximidade com a obtida pela enumeração exaustiva. A Figura 5 ilustra o desempenho do estimador em termos de módulo de tensão sem e com alocação de '4' medidores distribuídos na rede, respectivamente, onde se pode visualizar a melhoria no processo de EE (maior aproximação dos valores reais via FPC).

Tabela 6. Resultados SIA.

\begin{tabular}{lcccc} 
Sistema & 1 & 2 & 3 & 4 \\
\hline Localização & 25 & $24-26$ & $17-24-26$ & $7-14-24-26$ \\
FOB $(\%)$ & 7,5149 & 6,7408 & 5,6434 & 5,4285 \\
\hline
\end{tabular}

Tabela 7. Resultados Busca Exaustiva.

\begin{tabular}{lcccc} 
Medidores & 1 & 2 & 3 & 4 \\
\hline Localização & 25 & $24-26$ & $17-24-26$ & $7-14-24-26$ \\
FOB $(\%)$ & 7,5149 & 6,7408 & 5,6434 & 5,4285 \\
\hline
\end{tabular}

Destaca-se que em ambos os sistemas utilizados, o método proposto encontrou a mesma solução ou uma solução próxima ao método de enumeração exaustiva, porém, com tempos de simulação consideravelmente inferiores, conforme Tabela 8. Isto se deve fato da natureza combinatória 
do problema que, para o sistema de 33 barras, por exemplo, apresenta um total de combinações de 35.960 possíveis soluções para '4' medidores. Como o SIA efetua uma varredura mais eficiente do espaço de busca, determina soluções de boa qualidade sem avaliar todas as combinações, resultando na redução expressiva dos tempos de processamento.

Tabela 8. Tempo de simulação, 16 e 33 barras.

\begin{tabular}{lcccc} 
Medidores & 1 & 2 & 3 & 4 \\
\hline Localização (16) & 13 & $13-14$ & $2-13-14$ & $2-12-13-14$ \\
Tempo SIA (min) & 0,63 & 0,75 & 0,88 & 1,00 \\
Tempo Exaustivo (min) & 0,03 & 0,05 & 0,15 & 0,42 \\
\hline Localização (33) & 25 & $24-25$ & $17-24-25$ & $5-7-16-24$ \\
Tempo SIA (min) & 3,00 & 3,00 & 5,00 & 6,60 \\
Tempo Exaustivo (min) & 0,15 & 2,53 & 29,48 & 150,00 \\
\hline
\end{tabular}

\section{CONCLUSÃO}

Este trabalho apresentou um método de otimização inteira para determinar alocação ótima de medidores em redes de distribuição, visando máximo desempenho no processo de estimação de estados. Pôde-se concluir que os mecanismos inteligentes de busca da técnica utilizada permite uma investigação mais eficiente entre as soluções possíveis, evitando avaliar todas as combinações e, com isto, gerando soluções com significativo ganho de eficiência computacional. Além disto, a técnica proposta mantém uma boa qualidade das soluções, identificando a melhor global definida pela enumeração exaustiva ou alguma boa solução local ou subótima próxima da global.

\section{AGRADECIMENTOS}

Os autores deste artigo agradecem ao apoio da FAPEMIG, CNPq, CAPES, INERGE, e ao Grupo de Otimização GOPT.

\section{REFERÊNCIAS}

Abbasi, A.R. and Seifi, A.R. (2013). A new coordinated approach to state estimation in integrated power systems. International Journal of Electrical Power 8 Energy Systems, 45(1), 152 - 158.

Ahmad, F., Tariq, M., and Farooq, A. (2019). A novel ann-based distribution network state estimator. International Journal of Electrical Power $\&$ Energy Systems, 107, 200-212.

Baran, M.E. and Wu, F.F. (1989). Network reconfiguration in distribution systems for loss reduction and load balancing. IEEE Transactions on Power delivery, 4(2), 1401-1407.

Castro, P.A.D. (2009). Sinergia entre sistemas imunologicos artificiais e modelos graficos probabilisticos.

Chilard, O., Grenard, S., Devaux, O., and de Alvaro Garcia, L. (2009). Distribution state estimation based on voltage state variables: assessment of results and limitations. In CIRED 20th International Conference on Electricity Distribution. IET.

Civanlar, S., Grainger, J., Yin, H., and Lee, S. (1988). Distribution feeder reconfiguration for loss reduction. IEEE Transactions on Power Delivery, 3(3), 1217-1223.

De Castro, L.N. and Von Zuben, F.J. (2002). Learning and optimization using the clonal selection principle. IEEE transactions on evolutionary computation, 6(3), 239-251.
De Castro, L.N. and Von Zuben, F.J. (1999). Artificial immune systems: Part i-basic theory and applications. universidade estadual de campinas. Technical report, Dezembro de, Tech. Rep.

De Castro, L. and Von Zuben, F. (2000). Artificial immune systems: Part ii-a survey of applications. Technical report, Technical Report-RTDCA 02/00.

De Oliveira, L.W., de Oliveira, E.J., Gomes, F.V., Silva Jr, I.C., Marcato, A.L., and Resende, P.V. (2014). Artificial immune systems applied to the reconfiguration of electrical power distribution networks for energy loss minimization. International Journal of Electrical Power E Energy Systems, 56, 64-74.

Duque, F.G., de Oliveira, L.W., de Oliveira, E.J., and Augusto, A.A. (2017). State estimator for electrical distribution systems based on an optimization model. Electric Power Systems Research, 152, 122 - 129.

Gomez-Exposito, A. and Abur, A. (2004). Power system state estimation: theory and implementation. CRC press.

Korres, G. and Manousakis, N. (2012). State estimation and observability analysis for phasor measurement unit measured systems. IET generation, transmission \&6 distribution, 6(9), 902-913.

Majidi, M., Etezadi-Amoli, M., and Livani, H. (2017). Distribution system state estimation using compressive sensing. International Journal of Electrical Power \& Energy Systems, 88, 175 - 186.

Moradi-Sepahvand, M., Mashhour, E., and Mortazavi, S.S. (2019). Optimal placement of a combination of singlephase and three-phase $\mu$ pmus for observability of smart distribution networks with asymmetrical structure. International Journal of Electrical Power \& Energy Systems, 105, 592-601.

Oliveira, B.C.D. (2016). Estimação de estados em sistemas de distribuição: uma abordagem trifásica e descentralizada. Dissertação de mestrado, UFJF.

Qing, X., Karimi, H.R., and e Xingyu Wang, Y.N. (2015). Decentralized unscented kalman filter based on a consensus algorithm for multi-area dynamic state estimation in power systems. International Journal of Electrical Power \& Energy Systems, 65, 26 - 33.

Schweppe, F.C. and Rom, D.B. (1970). Power system static-state estimation. IEEE Transactions on Power Apparatus and Systems, (1), 125-130.

Singh, R., Pal, B., and Jabr, R. (2009). Choice of estimator for distribution system state estimation. IET generation, transmission \& distribution, 3(7), 666-678.

Sousa, P.H., Amaral, B.S., Oliveira, E., de Oliveira, L.W., and Oliveira, E.J. (2018). Sequential method of state estimation for distribution systems. In 2018 Simposio Brasileiro de Sistemas Eletricos (SBSE), 1-5.

Wang, H. and Schulz, N. (2004). A revised branch currentbased distribution state estimation algorithm and meter placement imapact. Transactions on Power Systems \& IEEE, 19, 207 - 2013.

Wood, A.J., Wollenberg, B.F., and Sheblé, G.B. (2013). Power generation, operation, and control. John Wiley $\&$ Sons.

Yang, X., Zhang, X.P., and Zhou, S. (2012). Coordinated algorithms for distributed state estimation with synchronized phasor measurements. Applied Energy, 96, 253 -260 . 